\title{
26 Research Square \\ Oil Palm Cultivation Critically Affects Sociality in an Endangered Malaysian Primate
}

\author{
Anna Holzner \\ Max Planck Institute for Evolutionary Anthropology \\ Krishna Balasubramaniam \\ University of California, Davis \\ Nadine Ruppert \\ Universiti Sains Malaysia \\ Anja Widdig ( $\square$ anja.widdig@eva.mpg.de ) \\ Max Planck Institute for Evolutionary Anthropology
}

\section{Research Article}

Keywords: Human, Oil, habitat, wildlife

Posted Date: December 21st, 2020

DOI: https://doi.org/10.21203/rs.3.rs-129385/v1

License: (9) This work is licensed under a Creative Commons Attribution 4.0 International License.

Read Full License 


\section{Abstract}

Human-induced habitat alterations globally threaten animal populations, often evoking complex behavioural responses in wildlife. This may be particularly dramatic when negatively affecting social behaviour, which fundamentally determines individual fitness and offspring survival in group-living animals. Here, we provide first evidence for critical behavioural modifications in sociality of Southern pigtailed macaques visiting Malaysian oil palm plantations in search of food. Specifically, we found significant reductions of positive social interactions, an increase of aggression and shifts in the macaques' social network structure, with the central positions of high-ranking adult females and immatures being passed to low-ranking individuals, likely resulting from socio-ecological risks posed by plantations. Deviations from natural behaviour also affected the smallest but crucial social units within groups, mother-infant pairs, with increased maternal protectiveness at plantations. We suggest that although primates can persist in human-altered habitats, their ability to adapt comes with a trade-off for their natural sociality, potentially hampering infant development and individual survival. Studies like ours remain critical for understanding species' adaptability to anthropogenic landscapes, which may ultimately contribute to facilitating their coexistence with humans and preserving biodiversity.

\section{Introduction}

The ongoing expansion of anthropogenic landscapes threatens rainforest ecosystems and the survival of many species ${ }^{1}$. Land conversion for food production and the cultivation of cash crops is the main driver for the global forest loss of estimated 10 million hectares per year ${ }^{2}$. Disturbing natural habitats and presenting sources of anthropogenic food, such modifications create novel and rapidly changing environments for animal populations ${ }^{3}$. With $60 \%$ of species being threatened ${ }^{4}$, non-human primates (hereafter 'primates') may be particularly susceptible to human disturbance. The Southern pig-tailed macaque (Macaca nemestrina, listed as Endangered by the IUCN ${ }^{4}$ ) has lost large parts of its natural forest habitat in Malaysia and Indonesia to oil palm monocultures ${ }^{5}$. Habitat fragmentation, hunting and conflicts with farmers are only some of the threats the macaques face in the forest-oil palm matrix 3,6 . Plantations also lack protection by dense forest vegetation and ease access for potential predators 3,7 . Largely understudied in the wild and described as elusive species that tend to avoid human contact ${ }^{6}$, it is imperative to better understand $M$. nemestrina's ability to adapt to these human-altered habitats.

Adaptive alterations in behaviour (i.e. behavioural plasticity ${ }^{8}$ ) are frequently one of the first visible responses of animals to human disturbance. In primates, these responses are diverse and very complex, with most previous studies focusing on their ability to exploit new feeding grounds, shifts in activity or ranging behaviour, and the negative consequences of the human-primate interface, such as increased stress levels among animals or conflicts over resources (reviewed in ${ }^{9}$ ). Despite their growing significance, quantitative studies on anthropogenic impact on wildlife behaviour have largely been context-specific and lack comparative assessments (reviewed in ${ }^{10}$ ). A few exceptional studies have focused on primates that live in (peri-)urban environments, describing substantial variation in time 
budgets ${ }^{11,12}$ and individual social behaviour ${ }^{13-15}$ across groups that face varying degrees of human disturbance.

In comparison, there is little in-depth knowledge of primate (and indeed wildlife) behaviour in agriculturally modified habitats. Particularly, differences in primate sociality between natural and anthropogenic habitats have not been systematically assessed. Yet, this is critical to understand as social behaviour plays a fundamental role in group-living animals. Macaques, as the majority of primate species, live in complex multi-male, multi-female societies, often following a dispersal regime, with males leaving their birth group to breed elsewhere, while females are philopatric and form the core of social groups ${ }^{16}$. Strong and enduring social bonds significantly increase fitness in both sexes, with social integration offering energetic benefits and buffering against social and environmental stress ${ }^{17,18}$. Specifically, the quality of affiliative relationships was found to predict individual reproductive performance ${ }^{19,20}$, longevity ${ }^{21}$, and infant survival ${ }^{22}$. Grooming is one of the most common affiliative interactions among primates, and has a key role in establishing and maintaining social relationships that underlie complex social features such as an animal's connectedness within the group's social network ${ }^{23-}$ 25 . Further, juvenile play constitutes a springboard for social competence during the first years of an individual's life ${ }^{26}$, allowing immatures to construct and expand their social networks and grow into their social roles as adults ${ }^{26,27}$. On the other hand, agonistic interactions are crucial in social groups, e.g. for the acquisition and maintenance of dominance status which directly impacts individual health ${ }^{28}$. Shifts in any of these behaviours, and (consequently) in individuals' social network roles, may impair social bonds and thus have downstream effects on group stability and survival ${ }^{29}$.

Though widely overlooked in previous research, behavioural plasticity in the smallest but most essential social units of a group, i.e. mother-infant pairs, may indicate how well a species can cope in humanaltered landscapes. Primate mothers provide extensive care to their offspring, and their behaviour strongly affects the development of a wide range of infant behaviours, including environmental exploration, affiliation and aggression, and later sexual and parental behaviour ${ }^{27,30}$. Depending on the social system of a species, but also on individual characteristics such as personality, dominance rank, parity, or infant age and sex ${ }^{31-34}$, mothering styles can vary from highly protective to highly tolerant, with the reduction of body contact and maternal permissive behaviours being particularly critical components for infant independence ${ }^{35}$. In this context, disruptions of the mother-infant relationship caused by habitat alterations may have severe consequences for offspring health and survival.

Macaca nemestrina frequently enter oil palm plantations which constitute an anthropogenically modified part of their natural range. Previous studies reported shifts in the macaques' foraging behaviour when ranging in these monocultures, complementing their diet with palm fruits and actively hunting plantation rats, an excellent source of protein ${ }^{36,37}$. Yet, it remains unclear whether and how the macaques' sociality in oil palm plantations deviates from their behaviour in their natural, undisturbed forest habitat. Here we take advantage of a 'natural experiment', by comparing the social behaviour of M. nemestrina in their natural habitat, the primary rainforest, with their behaviour observed in the human-altered plantation 
environment. Alternately ranging in these two different habitat types, the macaques serve as their own 'controls' in understanding how human disturbance can modify wildlife behaviour, ruling out the effects of group-specific factors, such as variation in group size, sex ratio, or cross-population genetic differences. Firstly, we provide an overview on the macaques' overall activity budget in forest and plantation habitat. Secondly, we examined quantitative differences in individuals' frequencies of affiliative and agonistic interactions across these habitats. Thirdly, we assessed potential differences in the macaques' social network connectedness. Finally, we investigated dynamics in the mother-infant relationship during the first six months after infant birth.

Based on previous findings $13,15,36$, we predicted strong shifts in time budgets across habitats, with macaques increasing foraging and feeding activity during plantation visits, while compromising on social interactions. We predicted rates of affiliation to be low at the risky plantation environment that lacks shelter and increases the visibility to predators ${ }^{3,7}$, but aggression to increase based on the assumption that plantations likely evoke stress in macaques and create competition for energy-rich food sources 11,38. In line with this, we predicted reductions in the number of individual interaction partners and the connectedness in social networks during plantation visits. Finally, we predicted macaque mothers to be more protective of their dependent offspring when ranging in the plantation compared to the forest.

\section{Results}

\section{The macaques' activity budget in forest and oil palm plantation}

We collected behavioural data on two habituated groups of macaques inhabiting the Segari Melintang Forest Reserve in Peninsular Malaysia and the surrounding oil palm plantation, with daily plantation visits lasting on average 2.9 hours (see Methods). As plantation areas in close proximity to the forests provide additional shelter and protection for animals through close-by forest vegetation, we distinguished between forest habitat, the plantation edge, i.e. plantation areas within 50 metres from the forest border, and areas further inside the plantation (hereafter 'plantation').

As a measure for socio-ecological risks posed by plantation and forest habitat, respectively, we recoded flight responses from potential predators shown by the macaques during our focal observations. Accounting for $83 \%$ of observed flight behaviour, humans and feral dogs were the most commonly observed threats. Both at the plantation edge and inside the plantation, flight responses from humans occurred at a rate of approximately 0.1 per hour observation time. In contrast, the rate of flight responses from humans was only 0.01 per hour in the forest. Similarly, flight from feral dogs was more frequently observed in the two plantation habitats ( 0.05 per hour) compared to the forest ( 0.01 per hour).

Overall, we found strong differences in the primates' time budgets across habitats (Fig. 1). Observed activities generally occurred in all three habitats, yet the rates at which they were performed remarkably changed when the macaques were ranging in the oil palm plantation. As predicted, approximately two 
thirds of the time the macaques spent at the plantation edge and inside the plantation, respectively, were dedicated to the search and consumption of food (mean \pm SD (plantation edge/plantation) $=0.68 \pm$ $0.08 / 0.65 \pm 0.08$ ). Socializing with conspecifics, in contrast, was drastically reduced inside the plantation (mean $\pm S D=0.003 \pm 0.006$ ) compared to the forest (mean $\pm S D=0.15 \pm 0.09$ ), yet it occurred at an intermediate rate at the plantation edge (mean $\pm S D=0.06 \pm 0.06)$.

\section{Social interactions in forest and oil palm plantation}

To gain a deeper insight into the macaques' social behaviour, we compared rates of affiliative and aggressive interactions between forest and plantation habitats. Accounting for $96 \%$ of the total time spent socializing, grooming and social play were the most commonly observed positive social interactions. During focal observations, we recorded a total of 1,607 grooming bouts and 574 bouts of juvenile social play. The rates of both grooming and social play significantly differed between habitats, while controlling for potentially confounding factors, particularly an individual's dominance rank and agesex class, the study group and time of the day (Likelihood ratio test (LRT, grooming/social play): $\chi^{2}=$ $120.96 / 40.28, \mathrm{df}=2, \mathrm{P}<0.001, \mathrm{~N}=1,535 / 510$ focal observations of $50 / 16$ individuals, details in Supplementary Table S1). Specifically, grooming rates were close to zero inside the plantation and significantly reduced at the plantation edge compared to the natural forest habitat (Fig. 2a). Similarly, social play was significantly higher in the forest than in both plantation habitats (Fig. 2b).

As predicted, differences in aggressive behaviour across habitats were generally in contrast to patterns of affiliation, although they were less clear, and varied according to the intensity of aggression. From a total of 496 observed aggressive interactions, 96 included physical aggression, while the others involved more ritualized forms, such as facial or vocal threats (hereafter 'non-physical aggression'). Whereas physical aggression was low in all three habitats and did not significantly differ between forest, plantation edge and plantation (LRT: $\chi^{2}=2.11, \mathrm{df}=2, \mathrm{P}=0.35, \mathrm{~N}=1,535$ focal observations of 50 individuals), nonphysical aggression was significantly higher inside the plantation compared to the forest and plantation edge (LRT: $\chi^{2}=6.06, d f=2, P=0.048, N=1,535$ focal observations of 50 individuals, Fig. 2c, Supplementary Table S1).

\section{Social network in forest and oil palm plantation}

In order to capture the impact of habitat alteration on complex patterns of an individual's social role that go beyond frequencies of social interactions, we examined differences in the macaques' social network connectedness when ranging at the oil palm plantation compared to the forest. To this end, we constructed social networks separately for each habitat based on dyadic affiliation which was measured as the proportion of point time scans two individuals were in social contact (i.e. grooming, social play or affiliative body contact) during behavioural observations. With only $0.2 \%$ of scans including positive social contact, dyadic affiliation was nearly absent inside the plantation. In contrast, $14.9 \%$ and $7.5 \%$ of scans in the forest and at the plantation edge, respectively, included affiliative contact. Therefore, further analysis was limited to describing differences in social network parameters between forest and plantation edge. 
We used binary degree as a measure of partner diversity, reflecting the overall number of social partners for each individual. This did not significantly differ between habitats, when controlling for individual dominance rank, age-sex class and the sampling effort ( $\chi^{2}=9.54$, $d f=8, P=0.30, N=36$ individuals). Mean partner diversities, i.e. the overall number of partners divided by the total number of proximity scans per individual, were 0.048 and 0.050 for forest and plantation edge, respectively.

We further investigated relative changes in individual scores of eigenvector centrality $(E C)$ between habitats, uncovering in how far an individual's position in its social group may differ between forest and plantation. EC accounts for both direct and indirect network ties, reflecting an individual's importance in the social network while considering the importance of its neighbours $29,39,40$. It was found to be strongly linked to indicators of individual health and fitness in group-living animals, including macaques ${ }^{23,40,41}$. Therefore, EC may be a 'proxy' behavioural (network) measure for our purpose of determining whether the effect of anthropogenic factors extend beyond time budgets, potentially impacting individuals' fitness and survival ${ }^{42,43}$. Additionally, we explored whether such differences in network positions might be dependent on an individual's socio-demographic attributes, particularly its dominance rank and age-sex class. In contrast to partner diversity, EC significantly differed between habitats as indicated by the LRT $\left(X^{2}=55.01, d f=8, P<0.001, N=36\right.$ individuals, details in Supplementary Table S2). To account for interdependency of network measures, particularly EC, we additionally ran node-swapping permutation tests ${ }^{44,45}$ which confirmed the significant effect of different habitats on EC (Supplementary Table S2). The significant three-way interaction between habitat, dominance rank and age-sex class indicates a clear, yet opposite, effect of dominance on EC in different habitats. Specifically, EC decreased with lower dominance in the forest, while it increased with lower dominance at the plantation edge (Fig. 3). In other words, high ranking individuals were better connected compared to lower ranking individuals when the group was ranging in the forest, while low-ranking individuals occupied the most central positions in the group at the plantation edge. This combined effect of dominance rank and habitat was found to be strongest for immature and adult females, moderate for immature males, and absent in adult males (Fig. 3).

\section{Mother-infant relationship in forest and oil palm plantation}

Studying affiliation between macaque mothers and their dependent offspring, as well as maternal behaviour encouraging infant independence during the first six months after infant birth, we found mothers' protectiveness to be strongly influenced by the habitat the macaques were ranging in.

Specifically, the proportion of body contact between macaque mothers and their offspring significantly differed between habitats, while controlling for infant age and sex, mothers' rank, parity and time of the day (LRT: $\chi^{2}=45.01, \mathrm{df}=4, \mathrm{P}<0.001, \mathrm{~N}=491$ observations of 11 mother-infant pairs, details in Supplementary Table S3). As predicted ${ }^{46}$ contact time decreased with infant age, yet the start of this decrease was highly dependent on the habitat (Fig. 4a). Specifically, body contact between mothers and infants already decreased within the first month after infant birth in the forest, after one to two months at the plantation edge, and only after approximately three months inside the plantation (Fig. 4a). 
Examining the mothers' incentive to facilitate infant independence, we looked at three different maternal behaviours, i.e. mother breaks contact, mother increases distance, and maternal rejection. With a total of only 15 occurrences during focal observations, rejection was very rare in macaque mothers and thus not considered for multivariate analysis. We further recorded 346 contacts broken by mothers and 838 events of mothers increasing the distance to their offspring. Infant age had a non-linear effect on both maternal behaviours, with the highest rates of breaking contact and increasing distance being observed at an age between three to five and four to six months, respectively (Fig. 4b, c). As indicated by the full-null model comparisons, the rates of both breaking contact and increasing distance, were significantly influenced by the habitat (LRT (breaking contact/increasing distance): $\chi^{2}=33.04 / 51.91, \mathrm{df}=6, \mathrm{P}<0.001, \mathrm{~N}=491$ observations of 11 mother-infant pairs, Supplementary Table S3). Specifically, the significant interaction between habitat and infant age indicates an earlier increase of mothers' facilitation of infant independence in the forest than in the plantation (Fig. 4b, c).

\section{Discussion}

The present study provides important insights into the effects of anthropogenic environments on primate social behaviour, which is crucial to understand a species' ability to coexist with humans. Our results demonstrate the presence of critical behavioural alterations in the macaques' activity while ranging in oil palm plantations, compromising on key social interactions, such as grooming and juvenile social play. Further, we found strong habitat-dependent shifts in the macaques' social network structure, with the central positions of high-ranking adult females and immatures of both sexes being passed to low-ranking individuals during plantation visits. Finally, we found dynamics in the mother-infant relationship, with mothers being more protective in the plantation compared to the forest as indicated by higher proportions of body contact and less maternal behaviour facilitating infant independence. Focusing on interindividual, between-context (forest versus oil palm plantation) variation in behaviour, we ensured a studydesign in which the study groups effectively formed their own 'controls' for our purposes of evaluating the clear effects of anthropogenic disturbance on spatial variation in aspects of animal behaviour that are critical to and underlie individuals' fitness.

Our results confirm previous studies, which suggest that oil palm plantations function as foraging grounds for macaques ${ }^{36,37,47}$. Their motivation to range within these monocultures despite increased predation risk, indicated by rates of flight responses five to ten times higher compared to those observed in the forest, likely lies in the broad abundance of food. Previous studies suggested more frequent plantation visits and extended plantation ranges during periods of lower fruit availability in the forest 47,48 . Further, regular plantation visits may be triggered by the high nutritional value of available food sources, as the macaques do not only feed on palm fruits, but also consume a high number of plantation rats ${ }^{37}$. Yet, highly clumped and energy-rich food sources may also increase competition and consequently fuel aggression among animals ${ }^{49,50}$, as previously shown for urban and semi-provisioned primate groups ${ }^{11,38}$. This was partly confirmed by our findings, with rates of non-physical aggression being significantly increased inside the plantation. However, no such effect was found for physical 
aggression, which in general is particularly rare in our study species, agreeing with $M$. nemestrina's intermediate position between despotic (i.e. less tolerant) and egalitarian (i.e. more tolerant) macaque species $^{34}$.

As predicted, rates of affiliative social interactions were rare in the oil palm plantation, especially when groups were ranging in plantation areas further than 50 metres from the forest border. Considering the socio-ecological risks posed by plantations (i.e. predation from feral dogs, human contact, and intense feeding competition within groups) the macaques may restrict plantation visits to feeding activities and avoid time-consuming social affiliations, which can be carried out more safely in the protected environment of the forest. As previously reported for other primate species, close proximity to humans and/or direct interactions with humans in touristic or urban areas can induce stress in animals ${ }^{15,51}$ and significantly alter affiliative interactions ${ }^{13-15}$. Our results add to these findings by showing that also indirect human impact through the conversion of rainforest into agricultural land can profoundly affect primate sociality. We found rates of both grooming and social play to be close to zero inside the plantation and significantly reduced at the plantation edge compared to the natural forest habitat. However, contrary to our prediction, the number of affiliative social partners did not differ between forest and plantation edge. This finding is nonetheless consistent with those by Marty et al. ${ }^{15}$, who revealed that long-tailed macaques ( $M$. fascicularis) living in human-impacted areas in Malaysia spent less time grooming but preserved the same number of grooming partners under time constraints. This retention of partner diversity is critical since both strong connections, i.e. frequent affiliative interactions, to an individual's favoured partners, but also a large number of weak connections to many different partners play an important role for individual fitness in Cercopithecine primates ${ }^{52}$.

Typically, animal social networks are influenced not only by individuals' demographic characteristics (e.g. age-sex class, dominance rank ${ }^{53,54}$ ), but also by extrinsic factors like their ecology (reviewed in ${ }^{55}$ ) or exposure to human impact ${ }^{43}$, with particularly the latter having been shown to decrease cohesiveness and connectivity of wildlife social networks ${ }^{42,56}$. Here we extend these previous findings, by revealing how environmental modifications, despite generating a seemingly uniform reduction in time spent socializing for entire groups (see above), may differentially affect the network connectedness of individual wild animals depending on their socio-demographic characteristics, using EC as a measure of social connectedness. In the forest, we observed a gradient in female centrality, with both high-ranking adult females as well as their immature female offspring being the most central, i.e. socially bestconnected individuals. This is consistent with previous studies, reporting top-ranking females to occupy more central network positions than lower-ranking individuals, as they are attractive social partners (e.g. by providing agonistic support in exchange for grooming ${ }^{57,58}$ ). Remarkably, at the planation edge, this relationship was reversed, possibly because high- and low-ranking females may use different strategies to handle the potentially stressful environment and intense feeding competition posed by the plantation. Reacting to time constraints, dominants may compromise on their affiliative social connections in order to be more vigilant of competing conspecifics. At the same time, subordinates may increase their affiliative network to gain more tolerance and/or access to energy-rich food sources from dominants. In 
immature males, we observed a similar, though less strong effect to that observed in females. This is unsurprising, as immature males are still integrated in the maternal network, holding their mothers' dominance status during childhood, yet they already start to grow into their later role as the dispersing sex ${ }^{27}$. In contrast, EC in adult males was not affected by rank and habitat, which may be closely linked to M. nemestrina's dispersal regime ${ }^{59}$. Whereas philopatric females strongly rely on being well integrated into an intact social network in order to survive and successfully raise offspring ${ }^{22}$, dispersing males have generally more peripheral positions within groups ${ }^{53}$.

Behavioural modifications in the groups' smallest social units can negatively affect both macaque mothers and their offspring. With mothers behaving more protective during plantation visits by increasingly keeping body contact, infant independence may be delayed compared to the macaques' natural behaviour in the forest. Yet, weaning ages in our study groups did not obviously deviate from the broader literature (N. Ruppert, unpublished data) which suggests that weaning in macaques occurs at about 10 to 14 months of age ${ }^{60}$. Additionally, alterations in mother-infant bonds may affect the development of infants in the forest-oil palm matrix. In particular, young males' preparation for their later dispersal may be hampered by prolonged physical proximity between mothers and infants. Further, increased maternal protectiveness may restrict adolescent females' opportunities to handle their adult relatives' infants, and thus to gain and practice maternal skills prior to their first own offspring 61,62. Ultimately, the disruption of an intact mother-infant relationship through frequent plantation visits may imply negative consequences for offspring health and survival. Long-term data (2014-2018) from our study groups, whose natural forest habitat have been partly replaced by oil palm plantation already several decades ago, revealed infant mortality within the first year of life to be approximately $55 \%$, with the highest rate (71\%) observed in 2016 (N. Ruppert, unpublished data). This is unexpectedly high, considering that infant mortality in other macaque species ranges between $2.7 \%$ and $32 \%{ }^{63-68}$. However, our data are not sufficient to prove whether infant survival is directly connected to the macaques' ability to cope with human-induced habitat modifications.

Overall, this study fundamentally contributes to understanding the impact of oil palm cultivation on sociality in wild primates. We observed behavioural plasticity in the macaques' overall network structure through to the smallest social units of the group, demonstrating that anthropogenic impacts, even without frequent direct contact with humans, can severely restrict affiliative interactions among macaques and disrupt the mother-infant relationship. High rates of infant mortality may ultimately cause difficulties for endangered species to maintain their viable population size. In this context, it is essential to protect the remaining populations and facilitate their coexistence with humans in anthropogenic landscapes. As umbrella species, primates represent a wide range of wildlife that depends on primary rainforest ${ }^{69}$. Hence, their protection will ultimately contribute to maintaining biodiversity and important ecosystem functions of tropical habitats. Unravelling the effects of both direct and indirect anthropogenic disturbances on primate social behaviour can serve as a basis for understanding the degree to which a species can adapt to human-altered habitats and may aid in developing effective conservation strategies and species management plans. Looking at the most important affiliative behaviours in primates, our 
results suggest that proximity to the forest is the key factor for macaques to be able to perform the full range of their natural behavioural repertoire. Maintaining forest corridors, an important conservation tool to create viable interfaces between forests and agricultural landscapes, may therefore not only facilitate natural dispersal and link fragmented wildlife populations affected by monocultures, but also enable animals to display essential social behaviours that directly affect their fitness. Ultimately, this will contribute to improving individual well-being and ensuring the long-term survival of primates and other species.

\section{Methods}

\section{Study site and subjects}

From January 2017 to September 2018, we collected non-invasive observational data on two habituated groups of wild Southern pig-tailed macaques (Macaca nemestrina) at the Segari Melintang Forest Reserve (SMFR) and the oil palm plantations bordering its south-western edge ( $\left.4^{\circ} 19-20^{\prime} \mathrm{N}, 100^{\circ} 34-36^{\prime} \mathrm{E}\right)$. To date, our study groups are the only wild population of this species that is successfully habituated, probably relating to the generally shy and elusive nature of $M$. nemestrina. The size and composition of the groups slightly changed during the study period, either due to male immigration or dispersal ${ }^{70}$, animals dying or being born, or juveniles reaching sexual maturity. During the study period, group 1 (named $A M Y$ ) consisted of 5 to 8 adult males, 12 to 15 adult females and 18 to 23 immature individuals. Group 2 (named VOL) consisted of 11 to 14 adult males, 19 to 21 adult females and 16 to 18 immature individuals. The age and sex of individual macaques were known from long-term observations ${ }^{36,37}$. Both groups visited the plantation area bordering their forest habitat almost daily (mean $\pm \mathrm{SD}(A M Y / V O L)=$ $3.1 \pm 1.8 / 2.7 \pm 1.8$ hours per day ${ }^{37}$ ). The annual home ranges of group $A M Y$ and $V O L$ were 92.7 and 96.6 hectares, respectively, with used plantation areas accounting for approximately one third of the total home range areas ${ }^{37}$. As group $V O L$ has not been fully habituated before the start of 2018 , assessments of the macaques' social network and the mother-infant relationship were performed only on group $A M Y$.

\section{Habitat types}

In order to assess the impact of anthropogenic environments on the macaques' social activities, we divided the home range areas of our study groups into three habitat types, i.e. forest, plantation edge and plantation (Supplementary Figure S1). SMFR comprises 2742 hectares of which 408 hectares are strictly protected Virgin Jungle Reserve. Its main vegetation types are dipterocarp lowland forest and alluvial fresh-water swamp ${ }^{36}$. The 420 -hectare sized oil palm plantation bordering the reserve was established between 1980 and 1990 and is managed by a federal authority. The oil palm estate was accessible to macaques, with encounters between wildlife and plantation workers being occasionally observed, yet these did not involve regular conflicts, such as hunting or chasing. We defined 'plantation edge' as the plantation area which is located within 50 metres from the forest border, whereas 'plantation' refers to all plantation areas further than 50 metres from the forest border. This distinction was made to account for the fact that plantation areas in close proximity to the forest provide additional shelter and protection for 
the macaques through close-by forest vegetation. So-called ecotones that form transitional areas between two distinct ecological habitats were reported to be of great environmental importance, potentially serving as speciation and biodiversity centres ${ }^{71}$. We chose the distance of 50 metres according to the average diameter of the macaque groups' dispersion (edge-centre-edge).

\section{Behavioural data collection}

We collected data using 30-minute focal animal sampling ${ }^{72}$ based on a species-specific ethogram established for the study species (adapted from ${ }^{73}$ ) in the forest, at the plantation edge and inside the plantation. We observed a total of 50 individually recognizable macaques (36 of group $A M Y, 14$ of group $V O L$ ). Focal individuals were chosen to represent all age-sex classes. The order of focal observations was randomized, aiming at sampling each individual only once per day. If a focal animal entered another habitat type during a 30-minute sampling protocol or went out of sight for more than ten minutes, this observation was stopped. Incomplete protocols were considered for multivariate analysis if they lasted at least 15 minutes. Total observation time was 724 hours (mean $\pm S D=14.5 \pm 3.6$ hours per subject).

To assess socio-ecological risks posed by forest and plantation habitats, we recorded the frequency and duration of flight behaviour shown by the macaques in response to the presence of potential predators, i.e. humans, feral dogs, birds of prey, snakes or monitor lizards. To provide an overview on shifts in the macaques' overall time budget, we continuously recorded their activity in the different habitats. Recorded activities included feeding (i.e. ingesting food), foraging (i.e. searching for or manipulating food), locomotion (i.e. walking, running, climbing or travelling without other activity), resting (i.e. standing, sitting or lying without other activity, eyes may be open or closed), socializing (i.e. all positive social interactions, e.g., grooming, being groomed and groom presenting, social play and huddling) and others (e.g. sexual and agonistic interactions or self-grooming). To assess individual differences in affiliation, we recorded the frequency, measured as rate per hour, and duration of all bouts of grooming and social play between the focal subject and other group members. As measures of frequency and duration were highly correlated (Pearson's $r$ (grooming/social play) $=0.78 / 0.81, P<0.001$ ), we considered only frequencies for analyses. Further, we recorded aggressive behaviour exchanged between the focal subject and other group members, considering both physical (i.e. attack, bite, grab, hit, push) and non-physical aggression (i.e. charge, chase, lunge, stare and vocal or open mouth threat). Social data were complemented by ad libitum data ${ }^{72}$ on aggression, displacement and submission among adult males and adult females for the purpose of constructing dominance hierarchies (see below). Data on social interactions included information on both the initiator and the recipient. Following previous studies ${ }^{74}$, a repetition of a behaviour was scored as a new bout if more than ten seconds had elapsed between occurrences or at least one partner had switched to a mutually exclusive activity (e.g., from grooming to aggression). During an aggressive event in which a number of different agonistic patterns occurred in quick succession, only the most intense kind of aggression was considered for analyses ${ }^{74}$.

To assess affiliative social networks across different habitats, we recorded data on spatial proximity between macaques. We took point time scans ${ }^{72}$ every three minutes within the 30 -minute sampling 
protocol, recording all group members in body contact with the focal individual. We further recorded whether or not this contact resulted from an affiliative interaction (e.g., during grooming, play or huddling). This was the case for $98.3 \%$ of our observations. The total number of scans recorded was 14,205 (mean $\pm S D=284 \pm 71$ scans per subject).

To assess the mother-infant relationship, we additionally observed eleven mother-infant pairs from group $A M Y$ in the three different habitats for the first six months after infant birth. Total observation time was 240 hours (mean $\pm S D=21.8 \pm 9.4$ hours per mother-infant pair). We continuously recorded maternal behaviour promoting infant independence ${ }^{46}$. Specifically, we recorded the number of contacts broken (i.e. any movements disrupting body contact between mother and infant), increases of distance (i.e. movements increasing the distance between mother and infant from within arm's reach (about $60 \mathrm{~cm})$ to outside of arm's reach) and maternal rejection (i.e. attempts by the infant to make contact that were rejected by the mother, e.g., by turning, running away, or holding the infant at a distance with an arm ${ }^{46}$ ). To ensure independence between these measures, increases of distance were only recorded if at least five seconds elapsed since contacts were broken. To assess spatial proximity in mother-infant pairs, we took point time scans ${ }^{72}$ every minute during focal observations, recording whether or not mothers and their infants stayed in body contact, including ventro-ventral contact, nipple holding, side-by-side contact, and grooming.

\section{Individual location data}

We collected individual location data with a Garmin GPSMAP62s daily during behavioural observations, with the coordinates of each focal observation being taken at half-time of the respective focal protocol. Annual home range areas of group $A M Y$ and $V O L$ were adopted from Holzner et al. ${ }^{37}$, showing point Kernel Density Estimations (KDE) with $95 \%$ probability of use ${ }^{75}$. To provide an overview on the occurrence of affiliative and aggressive social interactions across different habitats within the macaques' home ranges, we created interpolation maps (see Fig. 2) based on mean behavioural rates occurring during focal observations per $50 \mathrm{~m} \times 50 \mathrm{~m}$ grid cell using the Inverse Distance Weighting (IDW) tool of the software QGIS version 3.12 (QGIS Development Team 2020).

\section{Dominance hierarchy}

From 948 dyadic agonistic interactions with a clear winner and loser outcome collected during focal and ad libitum observations, we estimated rank orders using David's scores ${ }^{76}$. These were obtained in $\mathrm{R}$ version 3.4.4 (R Core Team 2020) using the function DS from the package 'EloRating' (version $0.46 .8{ }^{77}$ ). We set argument 'prop' to 'Dij', calculating dyadic win proportions corrected for chance ${ }^{78}$. As in macaques rank acquisition and function typically differ between sexes, with non-natal males fighting for dominance, while females socially inherit the rank of their mothers ${ }^{79}$, we estimated rank orders separately for males and females. Following Kaburu et al. ${ }^{80}$, we controlled for differences in group size and sex ratio by standardizing dominance rank as '(Rank-1)/(N-1)', where $\mathrm{N}$ represents the number of focal animals per group and sex class. Standardized dominance rank values range from zero (top- 
ranking individual) to one (bottom-ranking individual). Referring to literature ${ }^{81}$, immature males and females got assigned the same rank as their biological mother, or, if their mother already died, the rank of their closest adult female relative.

As David's scores do not account for potential temporal fluctuations in rank orders, we further assessed rank stability using the function stab.elo of the package 'EloRating' (version $0.46 .8{ }^{77}$ ). Indices range between zero (unstable) and one (stable), reflecting to what extent rank positions of individuals change over time. Males and females of both groups displayed stability indices close to one (males $(A M Y)=$ 0.9950 , females $(A M Y)=0.9956$, males $(V O L)=0.9909$, females $(V O L)=0.9943)$, suggesting highly stable dominance hierarchies during the sampling period in both sexes. Therefore, David's scores seem to be appropriate for estimating dominance ranks in our study groups.

\section{Social network analysis}

Based on affiliative interactions observed during individual focal sampling, we constructed the social network of group $A M Y$ separately for forest and plantation habitats. Following Lehmann et al. ${ }^{29}$, we assessed dyadic affiliation as the proportion of scans two individuals were in social contact (i.e. grooming, social play or affiliative body contact). We created social networks in $\mathrm{R}$ version 3.4.4 (R Core Team 2020) using an undirected data structure with the function graph_from_data_framefrom the package 'igraph' (version 1.2.5 ${ }^{82}$ ). For each individual, we extracted the binary degree and eigenvector centrality (EC), two commonly used network parameters to quantify individual social connectedness ${ }^{39,29}$. The binary degree reflects an individual's overall number of interaction partners, while EC is a measure of both direct and indirect network ties, reflecting a node's importance while considering the importance of its neighbours. Thus, a high value of EC suggests that an individual has many social partners who themselves also have many partners. While considering raw counts for binary degree, with regard to EC we were particularly interested in an individual's connectedness in relation to other group members. We therefore rescaled the obtained values of EC in each habitat to get percentile scores lying between zero (minimum) and one (maximum).

\section{Statistical Analysis}

Multivariate statistical analyses assessing the impact of different habitats on the frequencies of affiliation and aggression (models 1 to 4), social network connectedness (models 5 and 6 ), and the mother-infant relationship (models 7 to 9) were conducted in R version 3.4.4 (R Core Team 2020) using Generalized Linear Mixed Models (GLMM ${ }^{83}$ ). Models were fitted using the functions /merand g/mer of the package 'Ime4' (version 1.1.19 ${ }^{84}$ ) with the optimizer bobyqa. To facilitate model interpretation and convergence, we standardized all continuous predictors before model fitting to a mean of zero and a standard deviation of one ${ }^{85}$. Full-null model comparisons were derived using likelihood ratio tests (LRT ${ }^{86}$ ) using the R function anova with argument 'test' set to 'Chisq'. Tests of individual fixed effects were derived using the R function drop1 with argument 'test' set to 'Chisq', yet control predictors were not 
interpreted. Confidence intervals were derived using the function bootMer of the package 'Ime4' (version 1.1.19 ${ }^{84}$ ), using 1,000 parametric bootstraps.

Frequencies of affiliation and aggression (models 1 to 4): To investigate the impact of the habitat on the display of grooming, juvenile social play, and physical and non-physical aggression, we constructed four GLMMs ${ }^{83}$ with Poisson error structure and log link function. As response variables we used the number of grooming bouts (model 1), bouts of juvenile social play (model 2), bouts of physical aggression (model 3), and bouts of non-physical aggression (model 4) per focal observation $(\mathrm{N}=1,535$ focal observations of 50 individuals for models 1,3 and 4, and $N=510$ focal observations of 16 immature individuals for model 2). The models included the habitat (forest, plantation edge or plantation) as fixed effect test predictor, while controlling for individual dominance rank and age-sex class (adult male, adult female, immature male or immature female), as both rank and age-sex class have previously been shown to affect the occurrence of affiliative and agonistic interactions in macaques $27,57,87$. To account for changes in the overall group activity throughout the day, inter-group variation and repeated observations of the same individuals, we further included the time of the day, divided into four time blocks (early morning: 7:00-09:59 am, late morning: 10:00 am - 12:59 pm, early afternoon: 13:00-15:59 pm or late afternoon: 16:00-18:59 pm) and macaque group ( $A M Y$ or $V O L$ ) as fixed effect control predictors, and the focal individual ID and sampling date as random effects. Additionally, we included the random slopes of habitat and time of the day within focal individual in models 1 to 4 , and the random slope of rank within sampling date in models 1,3 and $4{ }^{86,88}$. Controlling for differences in the sampling effort, we further included the duration of each focal observation as an offset term into the models ${ }^{89}$. To test the effect of different habitats, we compared the full models with respective reduced models lacking only our test predictor (habitat) using a LRT ${ }^{86}$.

Social network connectedness (models 5 and 6): To investigate the impact of the habitat on two common network parameters, i.e. the binary degree and EC defined above, we constructed two GLMMs ${ }^{83}$ with Poisson and Gaussian error structure, respectively. As response variables we used the individuals' binary degree (model 5) and scaled EC (model 6) in each habitat ( $N=68$ observations of 34 individuals). We included the habitat (forest or plantation edge) and its interactions with individual dominance rank and age-sex class (as defined above) as fixed effects and the focal individual ID as random effect. Controlling for differences in the sampling effort, we further included the total number of point time scans per individual as an offset term into model $5^{89}$. To test the effect of different habitats on the binary degree and EC, we performed $\mathrm{LRTs}{ }^{86}$, comparing the full models with respective reduced models lacking our test predictor (habitat) and its interactions with dominance rank and age-sex class.

To account for interdependency of network measures used as outcome variables in our GLMMs, we used a node-swapping permutation approach, based on 1,000 permutations of the outcome variables ${ }^{90}$. This included recalculating the network parameters after randomly swapping the nodes of the original networks. We used node-swapping (as opposed to generating random graphs or using pre-network 'edgeswapping' randomizations) since this approach seemed better suited for our purposes of testing 
regression-based null hypotheses in a taxon with a largely stable group composition and relatively low observation biases 44,45 . Specifically, node-swapping preserves the overall size, number of connections, and structure of the network, thereby also preserving the overall distribution of node-level measures such as degree and EC. It is therefore a more conservative approach that may be less susceptible to Type I errors, compared to random graph generation or edge-swapping ${ }^{44,45}$. After each permuted swapping event, we refit the same GLMM using these newly created parameters as response variable. Comparing the observed model coefficients with the distribution of the 1,000 permuted coefficients, we calculated pvalues as the number of times the coefficient value of the observed network is smaller than a randomized network, divided by the number of randomizations ${ }^{90}$.

Mother-infant relationship (models 7 to 9): To investigate the impact of the habitat on the mother-infant relationship, we constructed three GLMMs ${ }^{83}$, with the proportion of body contact between mothers and offspring (model 7), the number of maternal breaks of contact (model 8), and the number of maternal increases of distance (model 9) per focal observation being the response variables ( $N=491$ focal observations of 11 mother-infant pairs for models 7-9). Investigating effects on the proportion of time spent in contact (model 7), we used a GLMM ${ }^{83}$ with binomial error structure and logit link function. In R, this analysis of proportions is possible by using a two-columns matrix with the number of contacts and non-contacts per individual as the response ${ }^{83}$. Models 8 and 9 were created using a count response with Poisson error structure and log link function. Here, we controlled for differences in the sampling effort by including the duration of each focal observation as an offset term ${ }^{89}$. In all three models, we included the habitat (forest, plantation edge or plantation) as a fixed effect test predictor, while controlling for infant and maternal characteristics, which were previously shown to affect the mother-infant bond, i.e. infant age ${ }^{46}$ and sex (male or female ${ }^{33}$ ), as well as maternal rank and parity (primiparous or multiparous ${ }^{31,32}$ ). As in models 1-4, we accounted for changes in the overall group activity over the day by including the time of the day as fixed effect control predictor. Further, we included the mother-infant pair and sampling date as well as the combination of these two as random effects, as mother-infant pairs were frequently observed more than once on a given day. Additionally, we included the random slopes of habitat, infant age and time of the day within the mother-infant pair ${ }^{86,88}$. As we expected infant age to have a non-linear effect on the rates of maternal breaking contact and increasing distance, we additionally incorporated squared infant age into models 8 and 9 . Further, we included the two-way interaction between habitat and infant age in model 7, and its interactions with infant age and squared infant age in models 8 and 9 . To test the effect of different habitats, we compared the full models with the respective reduced models lacking our test predictor (habitat) and its interactions with infant age and squared infant age, respectively, using LRTs ${ }^{86}$. In case of a non-significant interaction, we re-ran the model without the interaction term to facilitate the interpretation of the main effects in the model.

For models 1 to 9 , we assessed model stability by excluding the levels of the random effects one at a time and comparing the estimates from the obtained models with the estimates from the model based on all data using a self-written $\mathrm{R}$ function provided by Roger Mundry. This did not indicate any obviously influential case. To rule out collinearity, we determined Variance Inflation Factors (VIFs) for respective 
standard linear models excluding the random effects using the function vif of the package 'car' (version 3.0.2 ${ }^{91}$ ). According to these, none of the models indicated issues regarding collinearity (all VIFs $<1.16$ ). We confirmed that overdispersion was no issue in all models with a Poisson (models $1-4,5,8,9$ ) or binomial (model 7) error structure. Yet, there was an indication of underdispersion in model 3, probably reflecting the low rates of physical aggression in our dataset. Further, there was indication of slight underdispersion in models $7-9$, likely due to limited data points (dispersion parameters $=0.98 / 0.95$ / $0.45 / 0.77$ for models $1-4,0.95$ for model 5 and $0.65 / 0.67 / 0.52$ for models $7-9$ ). Visual inspections of a QQ-plot ${ }^{92}$ of the residuals and a scatterplot of the residuals plotted against the fitted values ${ }^{93}$ did not reveal obvious deviations from the assumptions of normally distributed and homogeneous residuals in the Gaussian model (model 6).

\section{Ethical note}

We obtained permits to study Macaca nemestrina from the Department of Wildlife and National Parks Peninsular Malaysia (permit holder: Dean of School of Biological Sciences, Universiti Sains Malaysia). We obtained permits to enter the forest reserve bordering the oil palm plantation from the Forestry Department Peninsular Malaysia (permit holder: Asyraf Mansor, School of Biological Sciences, Universiti Sains Malaysia). No written permit was needed to enter the plantations, but we informed the local management about the study. This non-invasive study was conducted in line with Universiti Sains Malaysia's animal ethics requirements.

\section{Declarations}

\section{Data availability.}

The data that support the findings of this study will be made available online.

\section{Acknowledgements}

We thank the Perak State Forestry Department and the Department of Wildlife and National Parks Peninsular Malaysia for research permits. We are very grateful to Antje Engelhardt, Asyraf Mansor and Shahrul Anuar Mohd Sah for their support in providing the necessary logistics during the pilot phase of this project. We also thank Brigitte Schlögl and Pascal Marty for valuable input on our statistical analysis, and Roger Mundry for providing R codes for model diagnostics. Special thanks to all students and volunteers who assisted in the field. The study was funded by USM Short-Term Grant (304/PBIOLOGI/6313256) and The Rufford Foundation UK (RSG 19842-2, both awarded to NR). We are also grateful to the Fundamental Research Grant Scheme (FRGS 203/PBIOLOGI/6711649), Disney Conservation Grant (through Malaysian Primatological Society), and The Habitat Foundation for supporting this ongoing long-term project (all to NR). Additional funding was provided through a PhD grant ('Doktorandenförderplatz' \# G00042) from the University of Leipzig as well as from the German Academic Exchange Service (DAAD) and the 'Gesellschaft für Primatologie' (GfP; all to AH). Finally, we thank the University of Leipzig and the Max-Planck Institute for the support of logistics. 


\section{Author Contributions}

Conceptualization, N.R., A.H. and A.W.; Methodology, A.H., N.R. and A.W.; Investigation, A.H.; Formal Analysis, A.H., K.N.B. and A.W.; Writing - Original Draft, A.H.; Writing - Review \& Editing, A.H., K.N.B., N.R. and A.W. Funding Acquisition, N.R. and A.H.

\section{Declaration of interests}

The authors declare no competing interests.

\section{References}

1. Rosa, I. M. D., Smith, M. J., Wearn, O. R., Purves, D. \& Ewers, R. M. The environmental legacy of modern tropical deforestation. Curr. Biol.26, 2161-2166 (2016).

2. FAO. Global Forest Resources Assessment 2020 - Key findings. Available at https://doi.org/10.4060/ca8753en (2020).

3. Estrada, A., Raboy, B. E. \& Oliveira, L. C. Agroecosystems and primate conservation in the tropics: A review. Am. J. Primatol.74, 696-711 (2012).

4. IUCN. The IUCN Red List of Threatened Species. Available at https://www.iucnredlist.org/en (2020).

5. Meijaard, E. et al.Oil Palm and Biodiversity: A Situation Analysis by the IUCN Oil Palm Task Force. (IUCN, Gland, 2018).

6. Bernstein, I. S. A field study of the pigtail monkey (Macaca nemestrina). Primates8, 217-228 (1967).

7. Azhar, B. et al. Contribution of illegal hunting, culling of pest species, road accidents and feral dogs to biodiversity loss in established oil-palm landscapes. Wildl. Res.40, 1-9 (2012).

8. Van Buskirk, J. Behavioural plasticity and environmental change. in Behavioural Responses to a Changing World: Mechanisms and Consequences (eds. Candolin, U. \& Wong, B. B. M.) 145-158 (Oxford University Press, 2012).

9. McLennan, M. R., Spagnoletti, N. \& Hockings, K. J. The implications of primate behavioral flexibility for sustainable human-primate coexistence in anthropogenic habitats. Int. J. Primatol.38, 105-121 (2017).

10. Balasubramaniam, K. N. et al. Impact of individual demographic and social factors on humanwildlife interactions: A comparative study of three macaque species. Sci. Rep.10, 21991 (2020).

11. el Alami, A., Lavieren, E. V., Rachida, A. \& Chait, A. Differences in activity budgets and diet between semiprovisioned and wild-feeding groups of the endangered Barbary macaque (Macaca sylvanus) in the Central High Atlas Mountains, Morocco. Am. J. Primatol.74, 210-216 (2012).

12. Koirala, S. et al. Diet and activity of Macaca assamensis in wild and semi-provisioned groups in Shivapuri Nagarjun National Park, Nepal. FPR88, 57-74 (2017).

13. Balasubramaniam, K. N. et al. Impact of anthropogenic factors on affiliative behaviors among bonnet macaques. Am. J. Phys. Anthropol.171, 704-717 (2020). 
14. Kaburu, S. S. K. et al. Interactions with humans impose time constraints on urban-dwelling rhesus macaques (Macaca mulatta). Behaviour156, 1255-1282 (2019).

15. Marty, P. R. et al. Time constraints imposed by anthropogenic environments alter social behaviour in long-tailed macaques. Anim. Behav.150, 157-165 (2019).

16. Schülke, O. \& Ostner, J. Ecological and social influences on sociality. in The Evolution of Primate Societies (eds. Mitani, J. C., Call, J., Kappeler, P. M., Palombit, R. A. \& Silk, J. B.) 193-219 (University of Chicago Press, 2012).

17. Young, C., Majolo, B., Heistermann, M., Schülke, O. \& Ostner, J. Responses to social and environmental stress are attenuated by strong male bonds in wild macaques. PNAS111, 1819518200 (2014).

18. McFarland, R. \& Majolo, B. Coping with the cold: Predictors of survival in wild Barbary macaques, Macaca sylvanus. Biol. Lett.9, 20130428 (2013).

19. Schülke, O., Bhagavatula, J., Vigilant, L. \& Ostner, J. Social bonds enhance reproductive success in male macaques. Curr. Biol.20, 2207-2210 (2010).

20. Kulik, L., Muniz, L., Mundry, R. \& Widdig, A. Patterns of interventions and the effect of coalitions and sociality on male fitness. Mol. Ecol.21, 699-714 (2012).

21. Silk, J. B. et al. Strong and consistent social bonds enhance the longevity of female baboons. Curr. Biol.20, 1359-1361 (2010).

22. Silk, J. B. et al. The benefits of social capital: Close social bonds among female baboons enhance offspring survival. Proc. R. Soc. B276, 3099-3104 (2009).

23. Brent, L. J. N., Lehmann, J. \& Ramos-Fernández, G. Social network analysis in the study of nonhuman primates: A historical perspective. Am. J. Primatol.73, 720-730 (2011).

24. Henzi, S. P. \& Barrett, L. The value of grooming to female primates. Primates40, 47-59 (1999).

25. Sueur, C., Jacobs, A., Amblard, F., Petit, O. \& King, A. J. How can social network analysis improve the study of primate behavior? Am. J. Primatol.73, 703-719 (2011).

26. Palagi, E. Not just for fun! Social play as a springboard for adult social competence in human and non-human primates. Behav. Ecol. Sociobiol.72, 90 (2018).

27. Amici, F., Kulik, L., Langos, D. \& Widdig, A. Growing into adulthood - a review on sex differences in the development of sociality across macaques. Behav. Ecol. Sociobiol.73, 18 (2019).

28. Vandeleest, J. J. et al. Decoupling social status and status certainty effects on health in macaques: A network approach. PeerJ4, e2394 (2016).

29. Lehmann, J., Majolo, B. \& McFarland, R. The effects of social network position on the survival of wild Barbary macaques, Macaca sylvanus. Behav. Ecol.27, 20-28 (2016).

30. Maestripieri, D. Maternal influences on primate social development. Behav. Ecol. Sociobiol.72, 130 (2018).

31. Maestripieri, D. Social and demographic influences on mothering style in pigtail macaques. Ethology104, 379-385 (1998). 
32. Fairbanks, L. A. Individual differences in maternal style: Causes and consequences for mothers and offspring. Adv. Stud. Behav.25, 579-611 (1996).

33. Kulik, L., Langos, D. \& Widdig, A. Mothers make a difference: Mothers develop weaker bonds with immature sons than daughters. PLoS One11, e0154845 (2016).

34. Thierry, B. Social epigenesis. in Macaque Societies. A model for the Study of Social Organization (eds. Thierry, B., Singh, M. \& Kaumanns, W.) 267-289 (Cambridge University Press, 2004).

35. Kaufman, I. C. \& Rosenblum, L. A. The waning of the mother-infant bond in two species of macaque. in Determinants of Infant Behavior (ed. Foss, B. M.) vol. IV 41-59 (Metheun, 1969).

36. Ruppert, N., Holzner, A., See, K. W., Gisbrecht, A. \& Beck, A. Activity budgets and habitat use of wild southern pig-tailed macaques (Macaca nemestrina) in oil palm plantation and forest. Int. J.

Primatol.39, 237-251 (2018).

37. Holzner, A. et al. Macaques can contribute to greener practices in oil palm plantations when used as biological pest control. Curr. Biol.29, R1066-R1067 (2019).

38. Ciani, A. C. Intertroop agonistic behavior of a feral rhesus macaque troop ranging in town and forest areas in India. Aggress. Behav.12, 433-439 (1986).

39. Bonnell, T. R., Vilette, C., Young, C., Henzi, S. P. \& Barrett, L. Formidable females redux: Male social integration into female networks and the value of dynamic multilayer networks. Curr. Zool. zoaa041 (2020).

40. Brent, L. J. N. Friends of friends: Are indirect connections in social networks important to animal behaviour? Anim Behav103, 211-222 (2015).

41. Balasubramaniam, K., Beisner, B., Vandeleest, J., Atwill, E. \& McCowan, B. Social buffering and contact transmission: Network connections have beneficial and detrimental effects on Shigella infection risk among captive rhesus macaques. PeerJ4, e2630 (2016).

42. Morrow, K. S., Glanz, H., Ngakan, P. O. \& Riley, E. P. Interactions with humans are jointly influenced by life history stage and social network factors and reduce group cohesion in moor macaques (Macaca maura). Sci. Rep.9, 20162 (2019).

43. Snijders, L., Blumstein, D. T., Stanley, C. R. \& Franks, D. W. Animal social network theory can help wildlife conservation. Trends Ecol. Evol.32, 567-577 (2017).

44. Farine, D. R. \& Carter, G. G. Permutation tests for hypothesis testing with animal social data: Problems and potential solutions. bioRxiv 2020.08.02.232710 (2020).

45. Weiss, M. N. et al. Common datastream permutations of animal social network data are not appropriate for hypothesis testing using regression models. Methods Ecol. Evol. 00, 1-11 (2020).

46. Maestripieri, D. Mother-infant relationships in three species of macaques (Macaca mulatta, $M$. nemestrina, $M$. arctoides). I. Development of the mother-infant relationship in the first three months. Behaviour131, 75-96 (1994).

47. Gazagne, E. et al. Northern pigtailed macaques rely on old growth plantations to offset low fruit availability in a degraded forest fragment. Am. J. Primatol.82, e23117 (2020). 
48. Caldecott, J. O. An Ecological and Behavioural Study of the Pig-Tailed Macaque. (S. Karger, 1986).

49. Kofron, C. P. Attacks to humans and domestic animals by the southern cassowary (Casuarius casuarius johnsonii) in Queensland, Australia. J. Zool.249, 375-381 (1999).

50. Uyeda, L. T., Iskandar, E., Kyes, R. C. \& Wirsing, A. J. Encounter rates, agonistic interactions, and social hierarchy among garbage-feeding water monitor lizards (Varanus salvator bivittatus) on Tinjil Island, Indonesia. Herpetol. Conserv. Biol.10, 753-764 (2015).

51. Behie, A. M., Pavelka, M. S. M. \& Chapman, C. A. Sources of variation in fecal cortisol levels in howler monkeys in belize. Am. J. Primatol.72, 600-606 (2010).

52. Ellis, S., Snyder-Mackler, N., Ruiz-Lambides, A., Platt, M. L. \& Brent, L. J. N. Deconstructing sociality: The types of social connections that predict longevity in a group-living primate. Proc. R. Soc. B286, 20191991 (2019).

53. Sosa, S. The influence of gender, age, matriline and hierarchical rank on individual social position, role and interactional patterns in Macaca sylvanus at 'La Forêt des Singes': A multilevel social network approach. Front. Psychol.7, 529 (2016).

54. Beisner, B. et al. A multiplex centrality metric for complex social networks: Sex, social status, and family structure predict multiplex centrality in rhesus macaques. PeerJ8, e8712 (2020).

55. Sueur, C., Romano, V., Sosa, S. \& Puga-Gonzalez, I. Mechanisms of network evolution: A focus on socioecological factors, intermediary mechanisms, and selection pressures. Primates60, 167-181 (2019).

56. Belton, L. E., Cameron, E. Z. \& Dalerum, F. Social networks of spotted hyaenas in areas of contrasting human activity and infrastructure. Anim. Behav.135, 13-23 (2018).

57. Schino, G. Grooming, competition and social rank among female primates: A meta-analysis. Anim. Behav.62, 265-271 (2001).

58. Wooddell, L. J., Kaburu, S. S. K. \& Dettmer, A. M. Dominance rank predicts social network position across developmental stages in rhesus monkeys. Am. J. Primatol.82, e23024 (2019).

59. Cords, M. Post-conflict reunions and reconciliation in long-tailed macaques. Anim. Behav. 44, 57-61 (1992).

60. Prescott, M. J., Nixon, M. E., Farningham, D. A. H., Naiken, S. \& Griffiths, M.-A. Laboratory macaques: When to wean? Appl. Anim. Behav. Sci. 137, 194-207 (2012).

61. Lancaster, J. B. Play-mothering: The relations between juvenile females and young infants among free-ranging vervet monkeys (Cercopithecus aethiops). Folia Primatol.15, 161-182 (1971).

62. Maestripieri, D. Social structure, infant handling, and mothering styles in group-living old world monkeys. Int. J. Primatol.15, 531-553 (1994).

63. Engelhardt, A. \& Perwitasari-Farajallah, D. Reproductive biology of Sulawesi crested black macaques (Macaca nigra). Folia Primatol.79, 326 (2008).

64. Takahata, Y. et al. Does troop size of wild Japanese macaques influence birth rate and infant mortality in the absence of predators? Primates39, 245-251 (1998). 
65. Krishna, B. A., Singh, M. \& Singh, M. Population dynamics of a group of lion-tailed macaques (Macaca silenus) inhabiting a rainforest fragment in the Western Ghats, India. Folia Primatol.77, 377-386 (2006).

66. Okamoto, K., Matsumura, S. \& Watanabe, K. Life history and demography of wild moor macaques (Macaca maurus): Summary of ten years of observations. Am. J. Primatol.52, 1-11 (2000).

67. Santosa, Y. Determination of long-tailed macaque's (Macaca fascicularis) harvesting quotas based on demographic parameters. Biodiversitas13, 79-85 (2012).

68. Fürtbauer, I., Schülke, O., Heistermann, M. \& Ostner, J. Reproductive and life history parameters of wild female Macaca assamensis. Int. J. Primatol.31, 501-517 (2010).

69. Marshall, A. J. \& Wich, S. A. Why conserve primates? in An Introduction to Primate Conservation (eds. Wich, S. A. \& Marshall, A. J.) 13-30 (Oxford University Press, 2016).

70. Greenwood, P. J. Mating systems, philopatry and dispersal in birds and mammals. Anim. Behav.28, 1140-1162 (1980).

71. Kark, S. Effects of ecotones on biodiversity. in Encyclopedia of Biodiversity (ed. Levin, S. A.) (Elsevier, 2013).

72. Altmann, J. Observational study of behavior: Sampling methods. Behaviour49, 227-267 (1974).

73. Thierry, B. et al. The social repertoire of Sulawesi macaques. Primate Res.16, 203-226 (2000).

74. Widdig, A., Nürnberg, P., Krawczak, M., Streich, W. J. \& Bercovitch, F. B. Affiliation and aggression among adult female rhesus macaques: A genetic analysis of paternal cohorts. Behaviour139, 371391 (2002).

75. Silverman, B. W. Density Estimation for Statistics and Data Analysis. Monographs on Statistics and Applied Probability. vol. 26 (Chapman and Hall, 1988).

76. David, H. A. Ranking from unbalanced paired-comparison data. Biometrika74, 432-436 (1987).

77. Neumann, C. et al. Assessing dominance hierarchies: Validation and advantages of progressive evaluation with Elo-rating. Anim Behav82, 911-921 (2011).

78. de Vries, H., Stevens, J. M. G. \& Vervaecke, H. Measuring and testing the steepness of dominance hierarchies. Anim. Behav.71, 585-592 (2006).

79. Bernstein, I. S. Dominance, aggression and reproduction in primate societies. J. Theor. Biol.60, 459472 (1976).

80. Kaburu, S. S. K. et al. Rates of human-macaque interactions affect grooming behavior among urban-dwelling rhesus macaques (Macaca mulatta). Am. J. Phys. Anthropol.168, 92-103 (2019).

81. Holekamp, K. E. \& Smale, L. Dominance acquisition during mammalian social development: The "inheritance" of maternal rank. Am. Zool.31, 306-317 (1991).

82. Csardi, G. \& Nepusz, T. The igraph software package for complex network research. Complex Syst.1695, 1-9 (2006).

83. Baayen, R. H. Analyzing Linguistic Data: A Practical Introduction to Statistics Using R. (Cambridge University Press, 2008). 
84. Bates, D., Mächler, M., Bolker, B. \& Walker, S. Fitting linear mixed-effects models using Ime4. J. Stat. Softw.67, 1-48 (2015).

85. Schielzeth, H. Simple means to improve the interpretability of regression coefficients. Methods Ecol. Evol.1, 103-113 (2010).

86. Barr, D. J., Levy, R., Scheepers, C. \& Tily, H. J. Random effects structure for confirmatory hypothesis testing: Keep it maximal. J. Mem. Lang.68, 255-278 (2013).

87. Kulik, L., Amici, F., Langos, D. \& Widdig, A. Sex differences in the development of aggressive behavior in rhesus macaques (Macaca mulatta). Int. J. Primatol.36, 764-789 (2015).

88. Schielzeth, H. \& Forstmeier, W. Conclusions beyond support: Overconfident estimates in mixed models. Behav. Ecol.20, 416-420 (2009).

89. McCullagh, P. \& Nelder, J. A. Generalized Linear Models. (Chapman and Hall, 1989).

90. Farine, D. R. \& Whitehead, H. Constructing, conducting and interpreting animal social network analysis. J. Anim. Ecol.84, 1144-1163 (2015).

91. Fox, J. \& Weisberg, S. An R Companion to Applied Regression. (Sage Publications, 2011).

92. Field, A. Discovering Statistics Using IBM SPSS Statistics. (Sage Publications, 2013).

93. Quinn, G. P. \& Keough, M. J. Experimental Design and Data Analysis for Biologists. (Cambridge University Press, 2002).

\section{Figures}
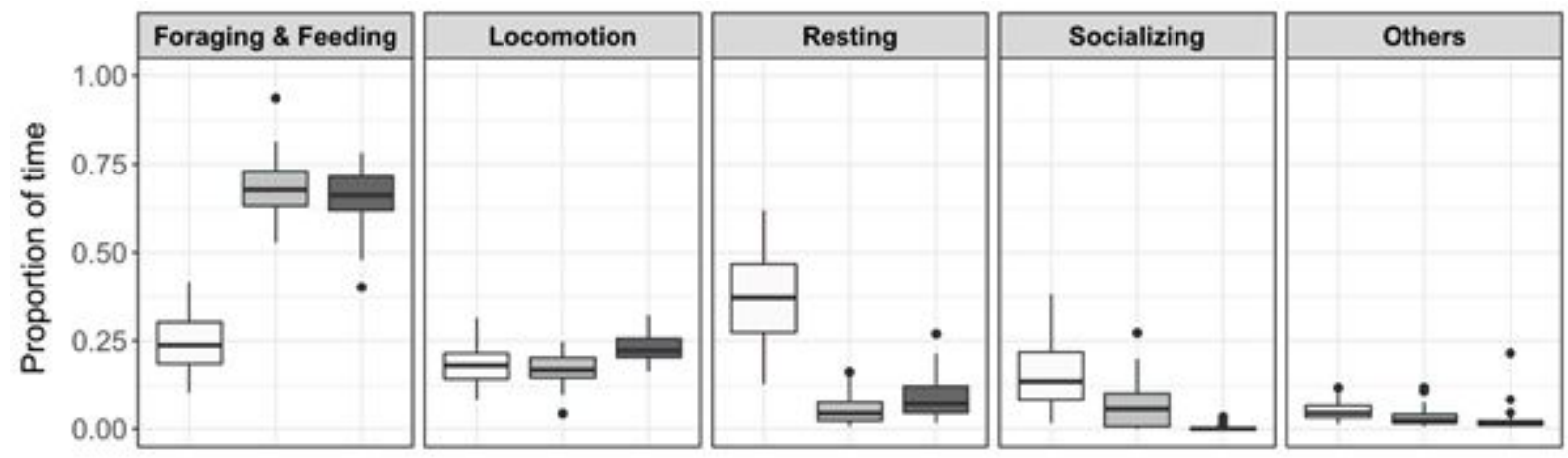

Habitat 官 Forest 追 Plantation edge

Plantation

\section{Figure 1}

Activity budgets of Southern pig-tailed macaques in forest and oil palm plantation. The boxplots indicate the median values and percentiles of individual proportions of time spent for foraging and feeding, locomotion, resting, socializing and other behaviours (e.g. self-directed behaviour, agonistic behaviour, mating), separately for forest, plantation edge and plantation. Circles represent outliers. The sample comprised a total of 50 individuals belonging to two social groups. 


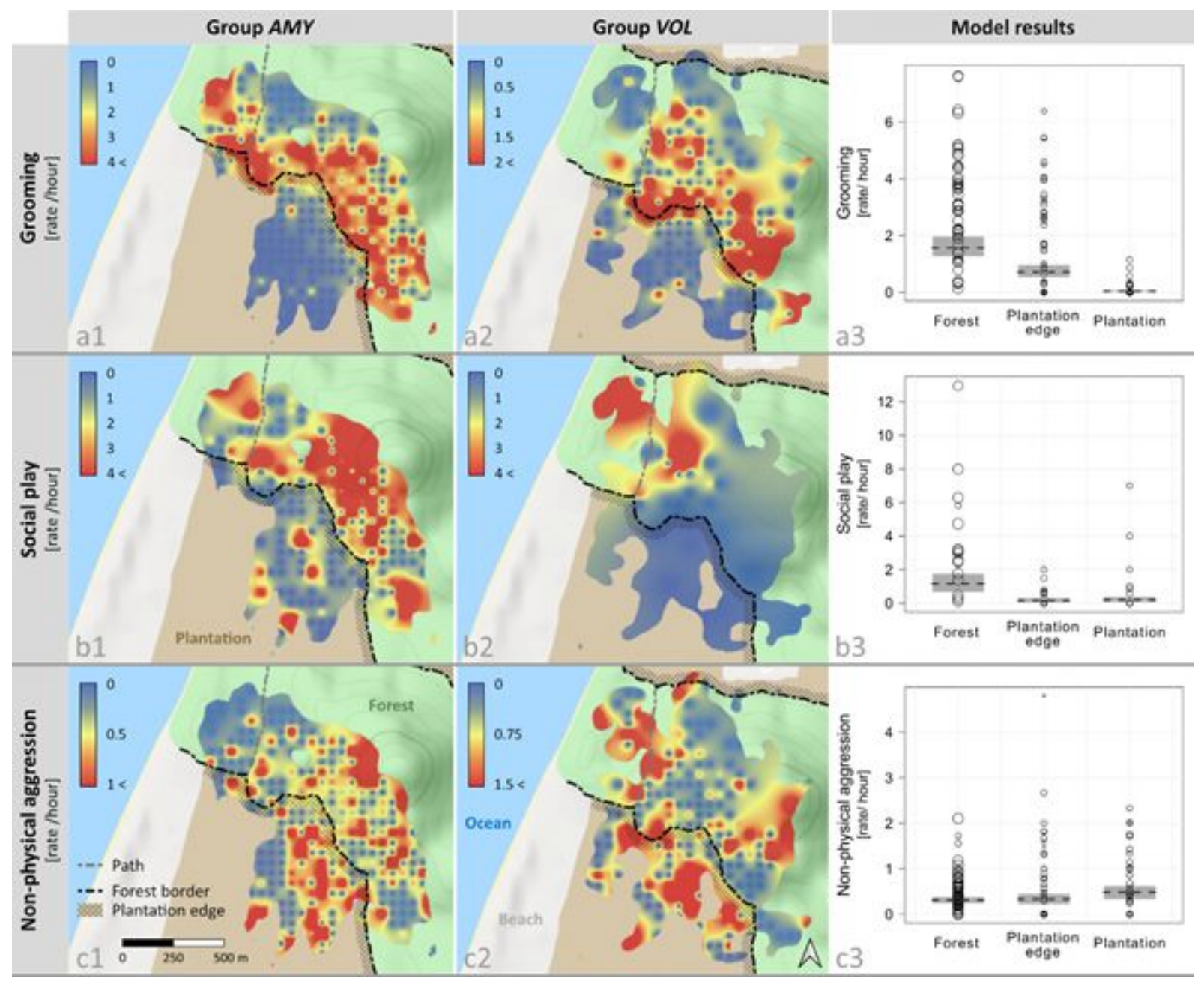

Figure 2

Affiliative and aggressive interactions among Southern pig-tailed macaques in forest and oil palm plantation. Interpolation maps indicate the mean rates per hour of grooming (a), juvenile social play (b) and non-physical aggression (c) occurring during focal observations per $50 \mathrm{~m} \times 50 \mathrm{~m}$ grid cell within the home range areas of group AMY (1) and group VOL (2). Model results (3) indicate predictions of the behavioural rates in the different habitats. Circles represent observation values, averaged per individual and habitat, with circle areas corresponding to respective sample sizes. The dashed lines show the fitted model and the shaded areas its $95 \%$ confidence intervals, conditional on rank being on its average, and based on age-sex class, group and time of the day manually dummy coded and then centred $(\mathrm{N}=1,535$ focal observations of 50 individuals (36 of AMY, 14 of VOL) for grooming and non-physical aggression and 510 focal observations of 16 individuals ( 14 of AMY, 2 of VOL) for social play). Note: The designations employed and the presentation of the material on this map do not imply the expression of any opinion whatsoever on the part of Research Square concerning the legal status of any country, territory, city or area or of its authorities, or concerning the delimitation of its frontiers or boundaries. This map has been provided by the authors. 

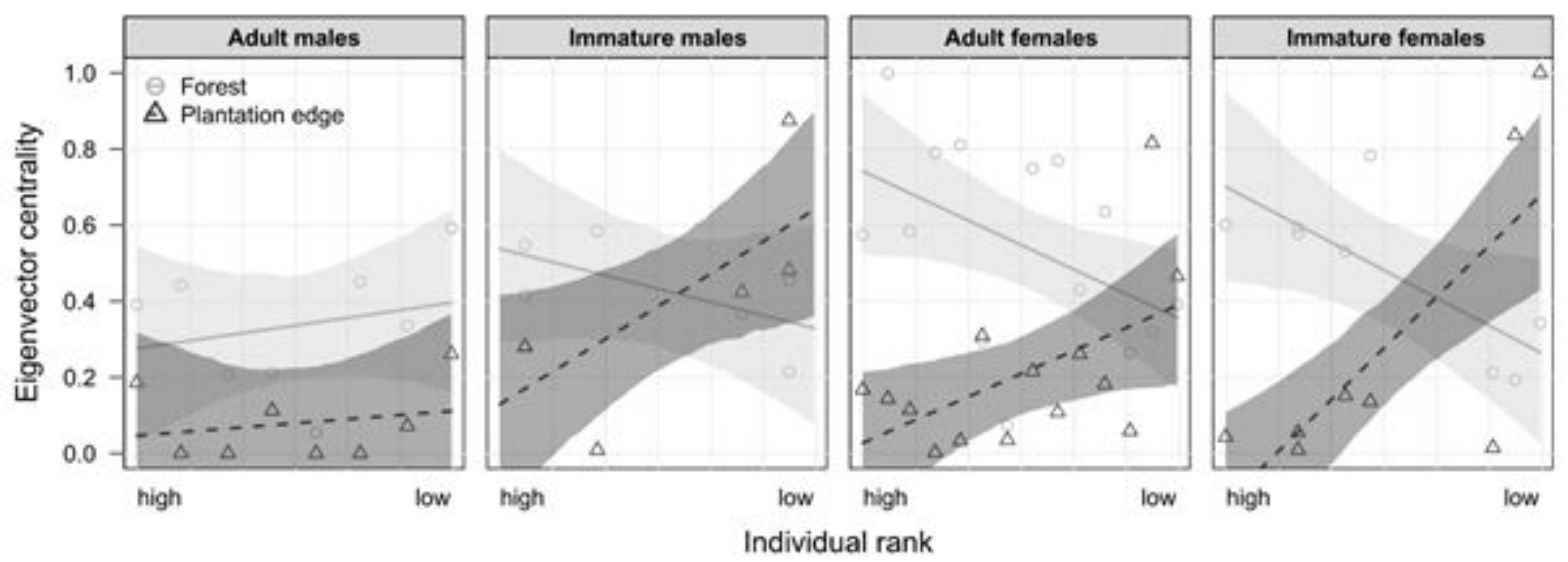

\section{Figure 3}

Macaques' social network roles in forest and oil palm plantation. Shown are individual scaled scores of eigenvector centrality as a function of individual rank, separately for forest and plantation edge, and for adult and immature males and females, respectively. The lines show the fitted model and the shaded areas its $95 \%$ confidence interval $(\mathrm{N}=36$ individuals). 

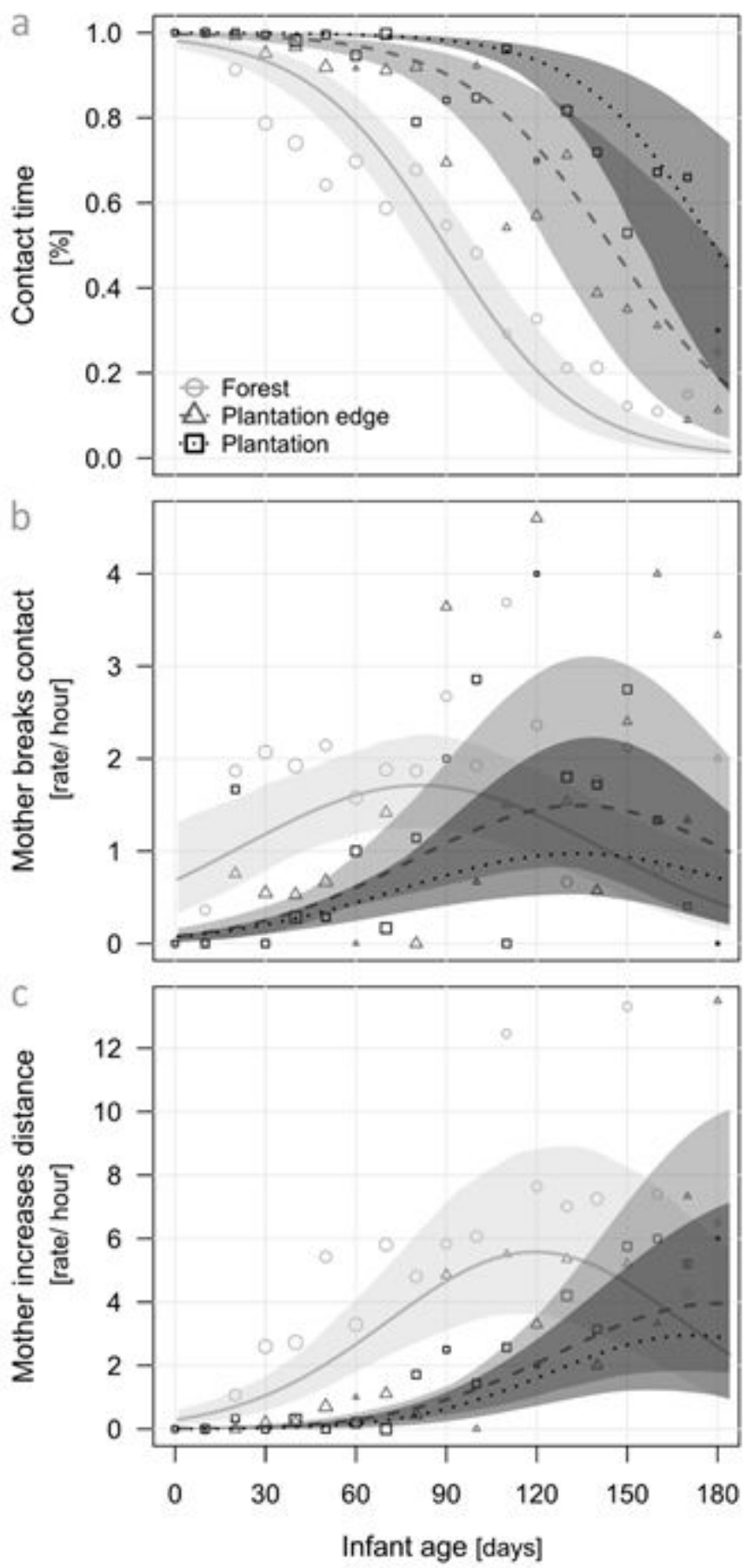

\section{Figure 4}

Mother-infant relationship in forest and oil palm plantation. Shown are the contact time between macaque mothers and their dependent offspring (a) and maternal facilitation of infant independence, measured as rates of breaking contact (b) and increasing distance (c), as a function of infant age, separately for forest, plantation edge and plantation. The lines show the fitted models, the shaded areas their $95 \%$ confidence intervals, conditional on continuous control predictors being on their average, and based on infant sex, parity and time of the day manually dummy coded and then centred. For visual clarity, infant age was binned into 10-day sections. The area of the points corresponds to the respective sample size (Total $\mathrm{N}=491$ focal observations of 11 mother-infant pairs). 


\section{Supplementary Files}

This is a list of supplementary files associated with this preprint. Click to download.

- SupplementSciRep.pdf 